\title{
Mild Trifunctional Protein Deficiency Is Associated with Progressive Neuropathy and Myopathy and Suggests a Novel Genotype-Phenotype Correlation
}

\author{
Jamal A. Ibdah, ${ }^{*}$ Ingrid Tein, ${ }^{\S}$ Carlo Dionisi-Vici," Michael J. Bennett, ${ }^{\natural}$ Lodewijk IJlst, ${ }^{\star \star}$ Beverly Gibson, ${ }^{\ddagger}$ \\ Ronald J.A. Wanders, ${ }^{* *}$ and Arnold W. Strauss ${ }^{\ddagger}$ \\ *Department of Medicine and ${ }^{\ddagger}$ Department of Pediatrics and Molecular Biology \& Pharmacology, Washington University School of \\ Medicine, St. Louis, Missouri 63110; ${ }^{\S}$ Departments of Pediatrics, Laboratory Medicine, and Pathobiology, University of Toronto, \\ Toronto, Ontario, Canada M5G 1X8; "Department of Metabolism, Bambino Gesù Children's Hospital, I-00165 Rome, Italy; "Department \\ of Pathology, University of Texas-Southwestern, Dallas, Texas 75235; and **Departments of Pediatrics and Clinical Chemistry, University \\ Hospital, 1105 AZ Amsterdam, The Netherlands
}

\begin{abstract}
Human mitochondrial trifunctional protein (TFP) is a heterooctamer of four $\alpha$ - and four $\beta$-subunits that catalyzes three steps in the $\beta$-oxidation spiral of long-chain fatty acids. TFP deficiency causes a Reye-like syndrome, cardiomyopathy, or sudden, unexpected death. We delineated the molecular basis for TFP deficiency in two patients with a unique phenotype characterized by chronic progressive polyneuropathy and myopathy without hepatic or cardiac involvement. Single-stranded conformation variance and nucleotide sequencing identified all patient mutations in exon 9 of the $\alpha$-subunit. One patient is homozygous for the T845A mutation that substitutes aspartic acid for valine at residue 246 . The second patient is a compound heterozygote for the T914A that substitutes asparagine for isoleucine at residue 269 and a $C 871 \mathrm{~T}$ that creates a premature termination at residue 255. Allele-specific oligonucleotide hybridization studies revealed undetectable levels of the mRNA corresponding to the mutant allele carrying the termination codon. This study suggests a novel genotype-phenotype correlation in TFP deficiency; that is, mutations in exon 9 of the $\alpha$-subunit, which encodes a linker domain between the $\mathrm{NH}_{2}$-terminal hydratase and the $\mathrm{COOH}$-terminal 3-hydroxyacyl-CoA dehydrogenase, result in a unique neuromuscular phenotype. (J. Clin. Invest. 1998. 102:1193-1199.) Key words: mitochondria $\bullet$ fatty acid metabolism $\bullet \beta$-oxidation $\bullet$ inborn error $\bullet$ neuropathy
\end{abstract}

\section{Introduction}

$\beta$-oxidation of fatty acids is the major source of energy for skeletal muscle and the heart, but the liver degrades fatty acids during prolonged fasting to generate ketone bodies. Mitochon-

J.A. Ibdah's current address is Department of Internal Medicine, Wake Forest School of Medicine, Wake Forest University, WinstonSalem, NC 27157.

Address correspondence to Arnold W. Strauss, Department of Pediatrics, St. Louis Children's Hospital, One Children's Place, St. Louis, MO 63110. Phone: 314-454-2284; FAX: 314-454-2476; E-mail: Strauss@kids.wustl.edu

Received for publication 24 October 1997 and accepted in revised form 29 July 1998.

J. Clin. Invest.

(C) The American Society for Clinical Investigation, Inc. 0021-9738/98/09/1193/07 \$2.00

Volume 102, Number 6, September 1998, 1193-1199

http://www.jci.org drial $\beta$-oxidation of long-chain fatty acids is a complex process requiring transport of the activated acyl-CoA moiety into the mitochondria and sequential removal of two-carbon, acetylCoA units. There are four reactions in the $\beta$-oxidation spiral mediated successively by an acyl-CoA dehydrogenase, a 2-enoyl-CoA hydratase, a 3-hydroxyacyl-CoA dehydrogenase, and a 3-ketoacyl-CoA thiolase. As the fatty acyl-CoA substrate becomes shorter, this spiral requires enzymes with different chain length specificities. To date, two 3-hydroxyacylCoA dehydrogenases have been identified to catalyze the third step: a long-chain 3-hydroxyacyl-CoA dehydrogenase (LCHAD) ${ }^{1}$ and a short-chain 3-hydroxyacyl-CoA dehydrogenase (SCHAD). This LCHAD activity lies within trifunctional protein (TFP), which is a heterooctamer of four $\alpha$ - and four $\beta$-subunits associated with the inner mitochondrial membrane $(1,2)$. The $\alpha$-subunit $\mathrm{NH}_{2}$-terminal domain contains the longchain 2-enoyl-CoA hydratase activity, and the $\mathrm{COOH}$-terminal domain contains the LCHAD activity. The $\beta$-subunit, which has the long-chain 3-ketoacyl-CoA thiolase activity, is encoded by a different gene. The human cDNAs encoding both subunits have been isolated and characterized $(3,4)$. We and others also defined the structure, chromosomal localization, and organization of both human TFP-subunit genes (3, $5)$. The $\alpha$-subunit gene contains 20 exons (3), and the $\beta$-subunit gene has 16 exons (5) (Sims, H.F., L. O'Brien, J. Fahrner, and A.W. Strauss, unpublished results). Both are present on chromosome 2p23 $(5,6)$ (Sims, H.F., L. O'Brien, J. Fahrner, and A.W. Strauss, unpublished results).

The emerging clinical significance of inherited disorders has greatly stimulated the study of mitochondrial $\beta$-oxidation. Enzymes and proteins essential for this pathway are encoded by nuclear genes, and defects are recessively inherited. LCHAD deficiency was first described in 1989 (7). Since that time, more than 100 LCHAD-deficient cases have been reported $(3,6-18)$. The rapid accumulation of patients suggests that LCHAD deficiency is, perhaps, the most common $\beta$-oxidation defect (8). Biochemical studies identified two groups of LCHAD-deficient patients (2, 3, 6-19). The first group has an "isolated" LCHAD deficiency (MIM 143450) with marked reduction in LCHAD activity but partial preservation of the activities of the other two TFP enzymes. The second group has complete TFP deficiency (MIM 251890), such that all three TFP enzyme activities are deficient $(2,5,10,11)$. Mutations in complete TFP deficiency have only been reported in five indi-

1. Abbreviations used in this paper: ASO, allele-specific oligonucleotide; LCHAD, long-chain 3-hydroxyacyl-CoA; SCHAD, short-chain 3-hydroxyacyl-CoA dehydrogenase; SSCV, single-stranded conformation variance; TFP, trifunctional protein; VLCAD, very long-chain acyl-CoA. 
viduals $(5,10,11)$. The majority of patients reported in the literature have isolated LCHAD deficiency. A limited number of reports describe the molecular defects in LCHAD deficiency $(3,6,7,12-14)$ and reveal that most patients carry at least one allele with the G1528C mutation in exon 15 of the $\alpha$-subunit that substitutes glutamate by glutamine (E474Q) in the protein.

TFP deficiency has a high mortality rate and is among the most severe of the fatty acid oxidation defects $(5,11)$. Typically, patients present acutely ill in infancy with a metabolic crisis (Reye-like syndrome), including hypoketotic hypoglycemia and hepatic encephalopathy that progresses to coma and death if not treated. Other early manifestations include cardiomyopathy or sudden, unexpected death. Surviving patients later often develop pigmentary retinopathy. Two manifestations of TFP or LCHAD deficiency are unique among the fatty acid oxidation disorders. First, three infants with hypocalcemia have been reported (15) (Ibdah, J.A., M.J. Dasouki, and A.W. Strauss, unpublished results). Second, mothers carrying fetuses with TFP or LCHAD deficiency may develop severe liver disease, including acute fatty liver of pregnancy or HELLP (hypertension, elevated liver enzymes, and low platelets) syndrome $(3,12)$.

An unusual, more chronic phenotype characterized by progressive sensorimotor polyneuropathy and limb-girdle myopathy with recurrent myoglobinuria has also been reported in some biochemically deficient patients (16-19). This phenotype has a later onset than most TFP- or LCHAD-deficient patients.

Correlation of mutations with phenotype has not been apparent in TFP or LCHAD deficiency (14). In this report, we describe two patients with TFP deficiency who presented with these unique neuromuscular manifestations. Molecular genetic analysis suggests a novel genotype-phenotype correlation.

\section{Methods}

Case history. The medical history of patient $\mathrm{A}$ has been previously described (16). Briefly, this 13-yr-old boy was born to asymptomatic first-cousin parents following a normal pregnancy and delivery. Gross motor milestones were delayed. At age $20 \mathrm{mo}$, he suffered the first of many episodes of profound weakness precipitated by fever, vomiting, or dehydration. His subsequent clinical course was characterized by a slowly progressive limb-girdle myopathy with mild facial weakness and a symmetrical peripheral sensorimotor axonopathy with secondary demyelination. In addition, the patient had recurrent episodes of myoglobinuria (up to five times per year) precipitated by prolonged exertion, infection, cold exposure, fasting, and/or stress. Muscle biopsy revealed a mild lipid-accumulative myopathy with intramyocardial lipid on electron microscopy. In the original reported assays (16), 3-hydroxyacyl-CoA dehydrogenase activity measured with longchain substrate $(\mathrm{C} 16)$ in skin fibroblast and muscle extracts was reduced. With the introduction of a frequent feeding, high-carbohydrate, low-fat diet and preventive fatty acid oxidation measures at age $7.5 \mathrm{yr}$, there was a marked reduction in the frequency of myoglobinuric episodes. However, no improvement in power or endurance occurred, and these continued to deteriorate. A trial of prednisone resulted in significant improvement in his limb-girdle myopathy, which has been sustained over $5 \mathrm{yr}$, as well as a transient improvement in his peripheral neuropathy. Myoglobinuric episodes were reduced to once every 1-2 yr and were less severe (16).

The clinical history of patient B was also reported previously (17). This now 12-yr-old boy was born to unrelated healthy parents, following an uneventful pregnancy and delivery. The first episode of muscle weakness at 13 mo of age was precipitated by an upper respiratory tract infection. Since then, the patient has experienced recurrent episodes of muscle weakness and myoglobinuria precipitated by infection, fasting, exertion, or cold exposure. At long-term follow-up, the patient had slow progressive sensorimotor polyneuropathy characterized by bilateral foot drop, contracture of the Achilles tendons, and symmetric weakness in wrist and finger extension. He does not have pigmentary retinopathy or cardiomyopathy. In the original report, 3-hydroxyacyl-CoA activity with long-chain substrate (C16) activity in skin fibroblast extracts was markedly reduced. A high-carbohydrate/ low-fat diet failed to prevent the progression of his neuromuscular manifestations. Long-chain 3-keto-acyl-CoA thiolase and hydratase activities were not previously reported in either patient.

Cell lines, enzymatic assays, and DNA, RNA, and protein isolation. Skin fibroblasts obtained from the patients were maintained in MEM supplemented with $10 \%$ FBS and $2 \mathrm{mM}$ glutamine. LCHAD and long-chain 3-ketoacyl-CoA thiolase activities were measured by the method of Wanders et al. (20). DNA from patient A's parents was isolated from white blood cells using alkaline lysis and proteinase digestion method (21). RNA was isolated using the acid guanidinium thiocyanate-phenol-chloroform extraction procedure (22). Protein was isolated from the fibroblasts using standard techniques (3).

Single-stranded conformation variance (SSCV) analysis of the $\alpha$-subunit. PCR was used to amplify the 20 exonic fragments of the $\alpha$-subunit in the presence of $\left[{ }^{32} \mathrm{P}\right] \mathrm{dCTP} .20$ sets of primers $(27-33 \mathrm{bp}$ in length) were designed so that the exons and 50-100 bp of flanking intronic sequences were amplified. These primers were designed based on sequence analysis of exon-intron junctions previously obtained in our laboratory (3). For amplification of exon 9, the following primer pair corresponding to intronic sequences flanking the exon were used: sense, 5'-GGGAATTCTAGGCTCTTTATAGTAAATATCT-3', and antisense, 5'-ATAGCAGAATTCAAGAAATTTAGTACTCAACA- $3^{\prime}$. After 5 min of denaturation at $95^{\circ} \mathrm{C}, 30$ cycles of PCR were performed using the following program: $30 \mathrm{~s} \mathrm{de}-$ naturation at $95^{\circ} \mathrm{C}, 30 \mathrm{~s}$ annealing at $55^{\circ} \mathrm{C}$, and $30 \mathrm{~s}$ extension at $72^{\circ} \mathrm{C}$. The amplified exonic fragments were analyzed by SSCV, using a nondenaturing gel according to the recommended protocol (23) except that electrophoresis was performed at $4^{\circ} \mathrm{C}$ for $8 \mathrm{~h}$ at $25 \mathrm{~W}$.

Allele-specific oligonucleotide (ASO) hybridization. Reverse transcriptase (RT) was used to synthesize cDNA from fibroblast RNA with oligo (dT) as the primer. This cDNA was then used in a first PCR reaction to amplify the full-length $\alpha$-subunit cDNA with the sense primer, 5' ${ }^{\prime}$-CTGCTGTCCTCTTCAGCTCAA- ${ }^{\prime}$, and antisense primer, 5'-ACTGACTGAGCGAGGCATGAG-3'. The second PCR reaction was "nested" using primers (sense, 5'-GAGACAAGGGATTGGTGGAAA-3' and antisense, 5'-GGCATCACTCCCTTGCTCAAT- $3^{\prime}$ ) that flank exon 9 coding sequences. The allele-specific oligonucleotide probes, complementary to the mutant or normal alleles (nucleotide differences bolded) were for patient A, mutant, 5'-CAACAGGATTACAAA-3' and normal, 5' -CAACAGGTTTA-

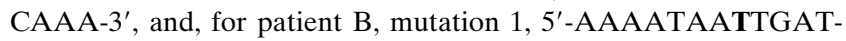
GTG-3' and normal 1, 5' ${ }^{\prime}$-AAAATAAATGATGTG-3' and mutation

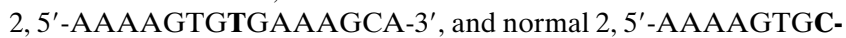
GAAAGCA. For ASO, $0.5 \mu \mathrm{g}$ of amplified RT-PCR product was applied to a nylon membrane with a vacuum blotting device. Hybridization with the ${ }^{32} \mathrm{P}$-labeled mutant or wild-type oligonucleotide was performed under stringent conditions (24).

Nucleotide sequence analysis. Direct sequencing of exonic fragments amplified from both genomic DNA or synthesized cDNA was performed using the appropriate sense or antisense primer. The PCR product was also placed in PCR-II vector, and nucleotide sequence analysis was obtained from 10-16 clones. For all mutations described in Results, sequences from subclones confirmed direct sequence data. Nucleotide sequencing was performed using the standard dideoxy chain termination method or with an automated sequencer and the Taq Dye Deoxy Terminator Cycle Sequencing Kit (Perkin-Elmer Corp., Norwalk, CT).

Northern and Western blot analyses. The integrity of RNA sam- 
ples were analyzed by formaldehyde gel electrophoresis and ethidium bromide staining. Northern blot analysis was performed using the $\alpha$-subunit ${ }^{32} \mathrm{P}$-cDNA probe hybridized under stringent conditions. Fulllength $\alpha$-subunit cDNA was isolated from a human heart library as described previously (3). Staining of transferred RNA with ethidium bromide was used to ensure uniform total cellular RNA recovery and transfer.

Western blot analysis was performed following $10 \%$ SDS-PAGE according to Laemmli (25) with rabbit polyclonal antibodies raised against the LCHAD domain of the $\alpha$-subunit and the entire $\beta$-subunit expressed in bacteria. For this bacterial expression, synthetic sense primers containing an artificial NdeI site corresponding to amino acid 304 of the $\alpha$-subunit and an antisense primer from the $3^{\prime}$ untranslated region with an engineered SalI site were used to amplify DNA from bp 1008-2316 with the entire $\alpha$-subunit cDNA as a template. This LCHAD domain encoding cDNA was placed into the NdeI and SalI sites of the expression plasmid, pet21a (Novagen, Madison, WI). After transformation into Escherichia coli and induction with isopropyl $\beta$-D-thiogalactoside for $4 \mathrm{~h}$, bacteria were lysed by sonication and subjected to centrifugation at $5,000 \mathrm{~g}$. The supernatant was analyzed by SDS gel electrophoresis, and the predominant stained band $\left(32,000\right.$ apparent $\left.M_{\mathrm{r}}\right)$ was excised from the gel and injected into rabbits as the LCHAD antigen. A similar strategy was used to express the complete, mature $\beta$-subunit with purification by SDS gel electrophoresis prior to antigen injection into rabbits. LCHAD antiserum diluted 1:2,000 and $\beta$-subunit antiserum diluted 1:3,000 were used on Western blots.

\section{Results}

Biochemical characterization. Table I shows the measured activities of four mitochondrial enzymes in extracts of cultured fibroblasts obtained from the two patients, as compared with normal controls. The patients both demonstrated TFP deficiency, with markedly reduced activities of both 3-hydroxyacyl-CoA activity versus long-chain (C16) substrate and longchain 3-ketoacyl-CoA thiolase. The $14-24 \%$ residual activity with the 3-keto-palmitoyl-CoA substrate is partially due to matrix $\operatorname{SCHAD}(5,26)$, which has an overlapping substrate specificity with TFP.

Delineation of the molecular defect in exon 9 of the $\alpha$-subunit. Our sequence data for the $\alpha$-subunit gene (3) were used to design 27-33 bp oligonucleotides to amplify each of the gene's 20 exons. The 20 exonic fragments amplified from the genomic DNA of the two affected children, the parents of pa-

Table I. Enzyme Activities in Cultured Skin

Fibroblast Extracts

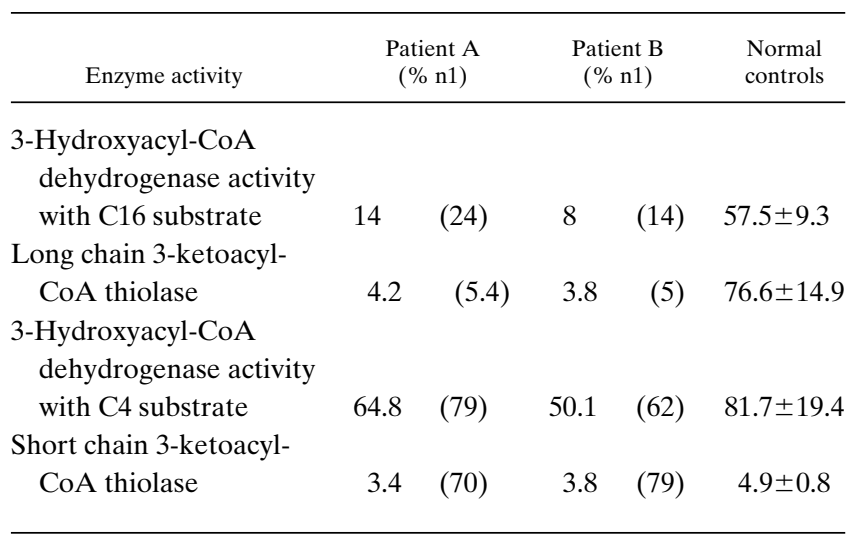

Values are in $\mathrm{nmol} / \mathrm{min} / \mathrm{mg}$ of protein with SD for control assays. tient $\mathrm{A}$, and a normal individual were screened for mutations by SSCV. Only amplified exon 9 for both patients and the parents of patient A revealed differences from normal (Fig. 1). Patient A (Fig. $1 A$ ) conformers reveal a two-band pattern, with both migrating differently than the two bands derived from a normal exon 9. This suggests a homozygous mutation in exon 9. The parents share identical patterns, with two normal and two aberrantly migrating bands, consistent with heterozygosity and their having the same mutation in exon 9. Direct nucleotide sequence of patient A's amplified exon 9 fragments showed that the affected child is homozygous for a single mutation, $\mathrm{T} \rightarrow \mathrm{A}$, at position 845 in the cDNA of the $\alpha$-subunit (Fig. 2 A). This mutation alters valine to aspartic acid at residue 246 of the mature $\alpha$-subunit. Both parents are heterozygous for the T845A mutation (Fig. 2 B).

Figure $1 B$ shows the SSCV analysis for patient $\mathrm{B}$. The patient's amplified exon 9 DNA exhibits four bands which are all different from normal, suggesting compound heterozygosity for two mutations. Direct sequence analysis of amplified genomic DNA confirmed compound heterozygosity (Fig. 3). The first mutation is $\mathrm{T} \rightarrow \mathrm{A}$ at position 914 in the cDNA of the $\alpha$-subunit (Fig. $3 A$ ). This mutation substitutes asparagine for
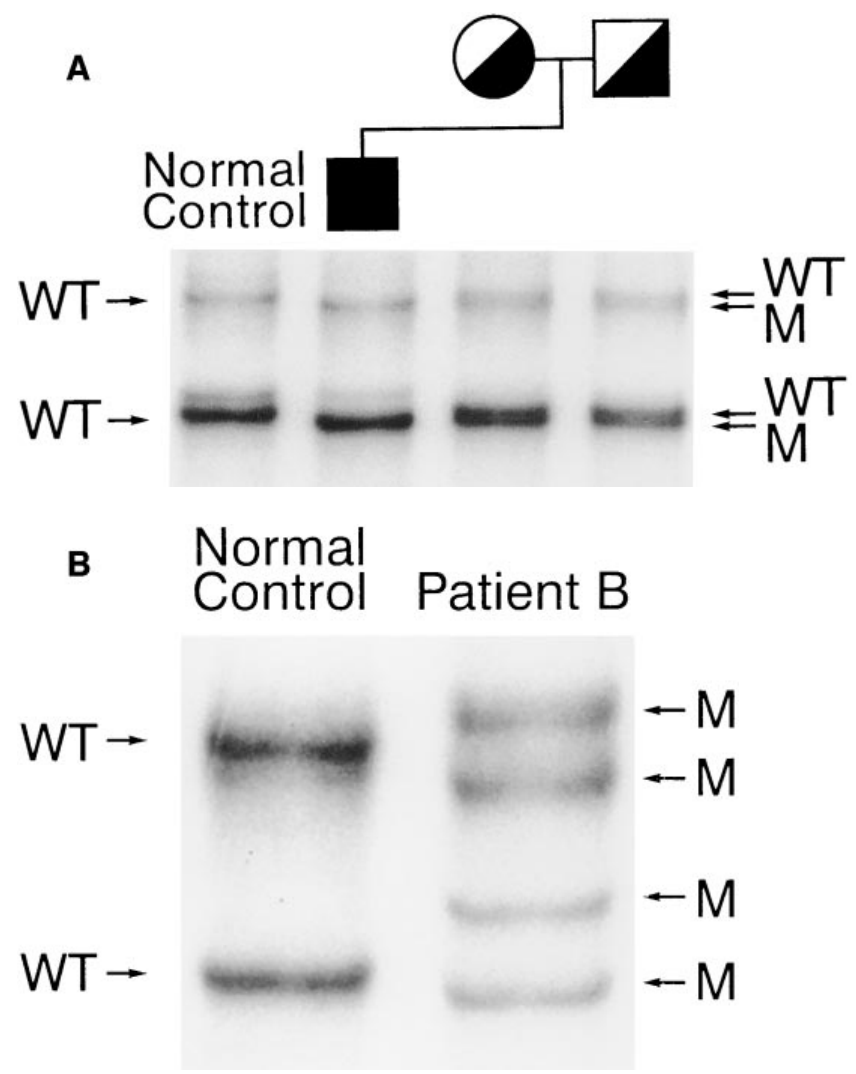

Figure 1. Exon $9 \mathrm{SSCV}$ analysis. (A) SSCV analyses of amplified exon 9 from a normal control (left lane), patient A (second lane), and the parents. The family pedigree is shown above the gel photograph. The " $\leftarrow$ WT," an abbreviation for wild type, indicates bands derived from normal alleles. The " $\leftarrow \mathrm{M}$ " designates bands from the mutant allele. (B) SSCV analyses for patient B (right lane) and a normal control (left lane) with the bands labeled as in A. Amplification of exon 9 genomic DNA from the various individuals was performed as described in the Methods section and analyzed by SSCV gel electrophoresis (23). 


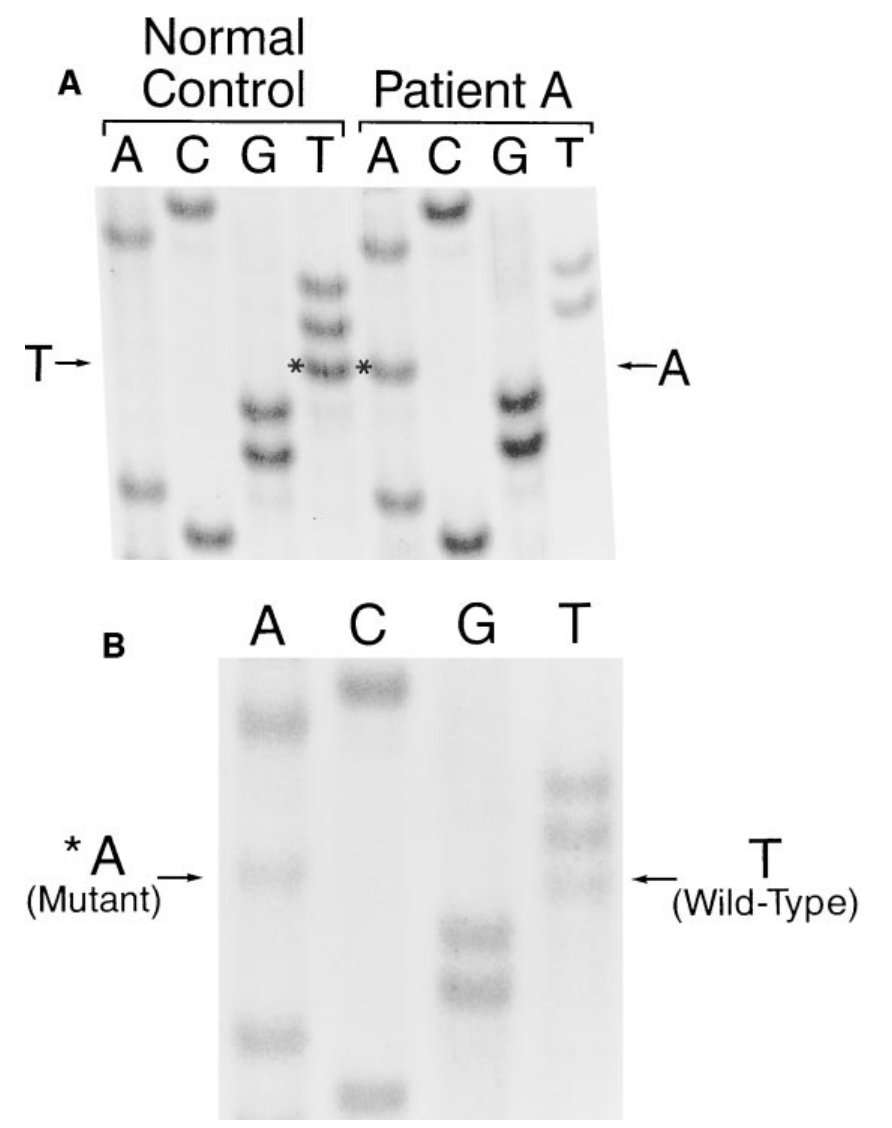

Figure 2. Delineation of exon 9 mutation in family A. (A) Direct sequence analysis of amplified exon 9 genomic DNA from patient $\mathrm{A}$ and normal control. The patient is homozygous for an A at bp 845 of the $\alpha$-subunit coding region. The normal sequence at bp 845 is $T$, the second base of a valine codon. The mutation T845A substitutes aspartic acid for valine at amino acid 246 of the mature TFP $\alpha$-subunit. $(B)$ Direct sequence analysis of amplified exon 9 genomic DNA from the mother of patient $\mathrm{A}$. The sequence contains both $\mathrm{T}$ and $\mathrm{A}$ at position 845 , indicating heterozygosity for this mutation. The father is also heterozygous for this mutation (data not shown). The asterisks indicate the site of the mutations in the sequence ladder.

isoleucine at residue 269 in the mature $\alpha$-subunit. The second mutation is $\mathrm{C} \rightarrow \mathrm{T}$ at position 871 in the cDNA of the $\alpha$-subunit (Fig. 3 B). This mutation creates a stop codon (UGA) and would cause premature termination at residue 255 of the mature $\alpha$-subunit. Parental DNA was not available for testing. We conclude that both patients with the progressive neuromuscular phenotype have mutations in the TFP $\alpha$-subunit exon 9.

Confirmation of exon 9 mutations by ASO hybridization. Amplified genomic exon 9 fragments from patient $\mathrm{A}$ and a normal control were probed separately with two different oligonucleotides carrying the T845A mutation or the corresponding normal sequence. The mutant probe hybridized only with the DNA from patient A, while the normal probe hybridized only with the DNA from the normal control (data not shown). This confirms homozygosity for this mutation in patient A. Similarly, amplified genomic exon 9 fragments from patient B and normal control were hybridized separately to oligonucleotides carrying the C871T or T914A mutations and the corresponding normal sequences. The mutant and normal probes
A Patient $B \quad$ Normal
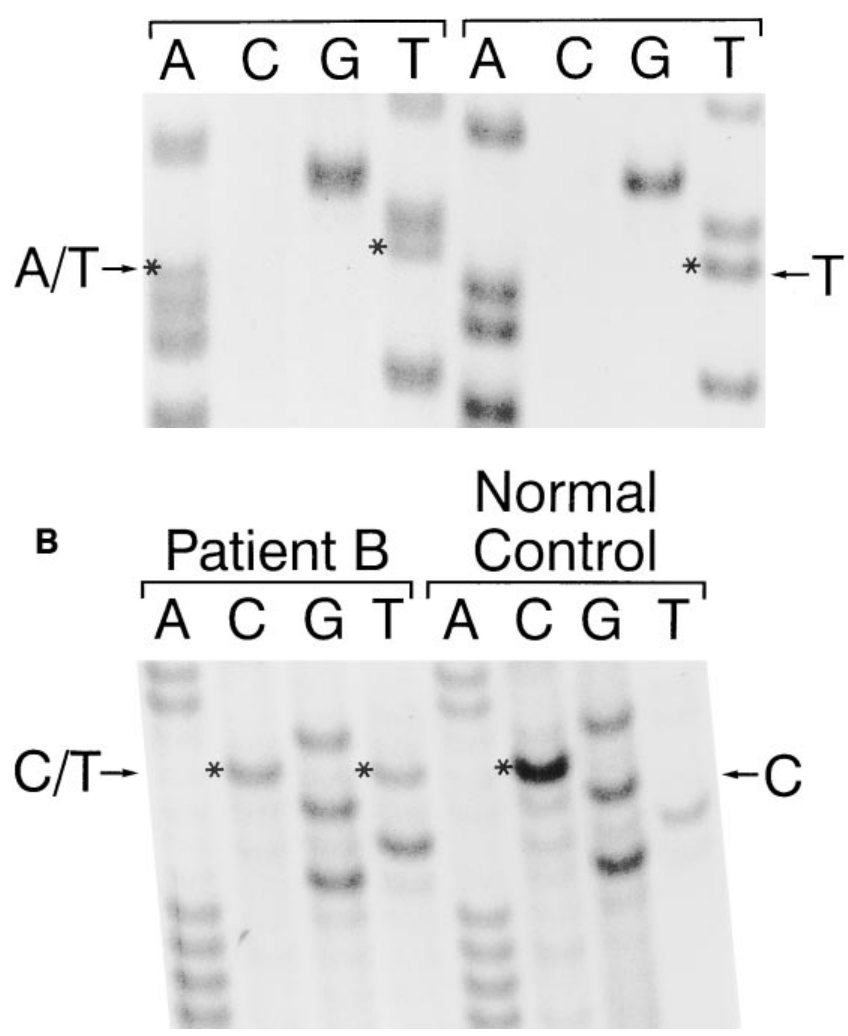

Figure 3. Delineation of exon 9 mutations in patient B. Direct sequence analysis of amplified exon 9 genomic DNA from patient $\mathrm{B}$ compared with a normal control. (A) T914A mutation. The normal sequence at bp 914 is $\mathrm{T}$, the second base of the isoleucine codon at amino acid 269. The mutation is $\mathrm{A}$, the second base of an asparagine codon. The patient's sequence contains both $\mathrm{T}$ and $\mathrm{A}$ at position 914, indicating heterozygosity for this mutation. $(B)$ C871T mutation. The normal sequence at bp 871 is $C$, the first base of an arginine codon. The mutation is $\mathrm{T}$, the first base of a termination codon. The patient's sequence contains both $\mathrm{C}$ and $\mathrm{T}$ at position 871 , indicating heterozygosity for this mutation. The asterisks indicate the site of the mutations in the sequence ladder.

hybridized with the DNA from patient B, while only the normal probes hybridized with the DNA from normal control (data not shown). Densitometric scanning revealed that the ratio of hybridization of the normal probe to the DNA from patient B was half that of the normal control. These results confirm compound heterozygosity of patient B for the C871T and T914A mutations.

Delineation of the molecular defect in exon 9 at the $m R N A$ level. Northern blot analysis of total RNA isolated from cultured skin fibroblasts and quantification by densitometric scanning revealed that patient $\mathrm{A}$ had normal expression of $\alpha$-subunit mRNA ( $89 \%$ of control), but that patient B had reduced expression ( $56 \%$ of control). To characterize further the expressed mRNA, RT-PCR was used to synthesize and amplify $\alpha$-subunit mRNA cDNA encompassing the exon 9 region. Direct sequencing of the cDNA fragment revealed that all detectable, expressed mRNA in patient A contained the T845A mutation (data not shown), consistent with homozygosity. The expressed mRNA of patient B contained only the 
T914A mutation. No amplified cDNA with the second mutation, C871T (255ter) was detected (data not shown). Together, the Northern blot and RT-PCR results indicate that the cytoplasmic mRNA transcript carrying the premature termination codon mutation is not stably expressed at detectable levels.

To confirm this observation and to quantify allele-specific mutant mRNA expression, ASO hybridization using the probes described above was performed on amplified cDNA fragments encompassing exon 9 obtained from the patients' and normal control cultured fibroblasts (Fig. 4). The probe carrying the T845A mutation hybridized only to the cDNA of patient $\mathrm{A}$, and the probe carrying the corresponding normal sequence hybridized only to the normal control (Fig. $4 \mathrm{~A}$ ). Similarly, the probe carrying the T914A mutation hybridized only to the cDNA from patient $\mathrm{B}$, and the probe carrying the corresponding normal sequence hybridized only to the normal control (Fig. 4 B, bottom). In contrast, the cDNA fragments from patient $\mathrm{B}$ and the normal control did not hybridize with the probe carrying the stop codon mutation (C871T), but both hybridized with the probe carrying the corresponding normal sequence (Fig. 4 B, top). Densitometric scanning revealed that hybridization of the probe carrying the $\mathrm{C} 871 \mathrm{~T}$ mutation to the cDNA of patient $\mathrm{B}$ is only minimally above background and $<3 \%$ of the corresponding normal probe binding to the cDNA from the normal control.

The above results indicate stable expression of the mRNAs containing the T845A and the T914A missense mutations in patients $\mathrm{A}$ and $\mathrm{B}$, respectively. In contrast, there was no detectable expression of mRNA from the allele carrying the premature termination codon mutation $(\mathrm{C} 871 \mathrm{~T})$ in patient $\mathrm{B}$.

Western blot analysis. To determine the level of expression of TFP subunits in the patients' fibroblasts, we performed Western blot analysis with rabbit polyclonal antibodies raised

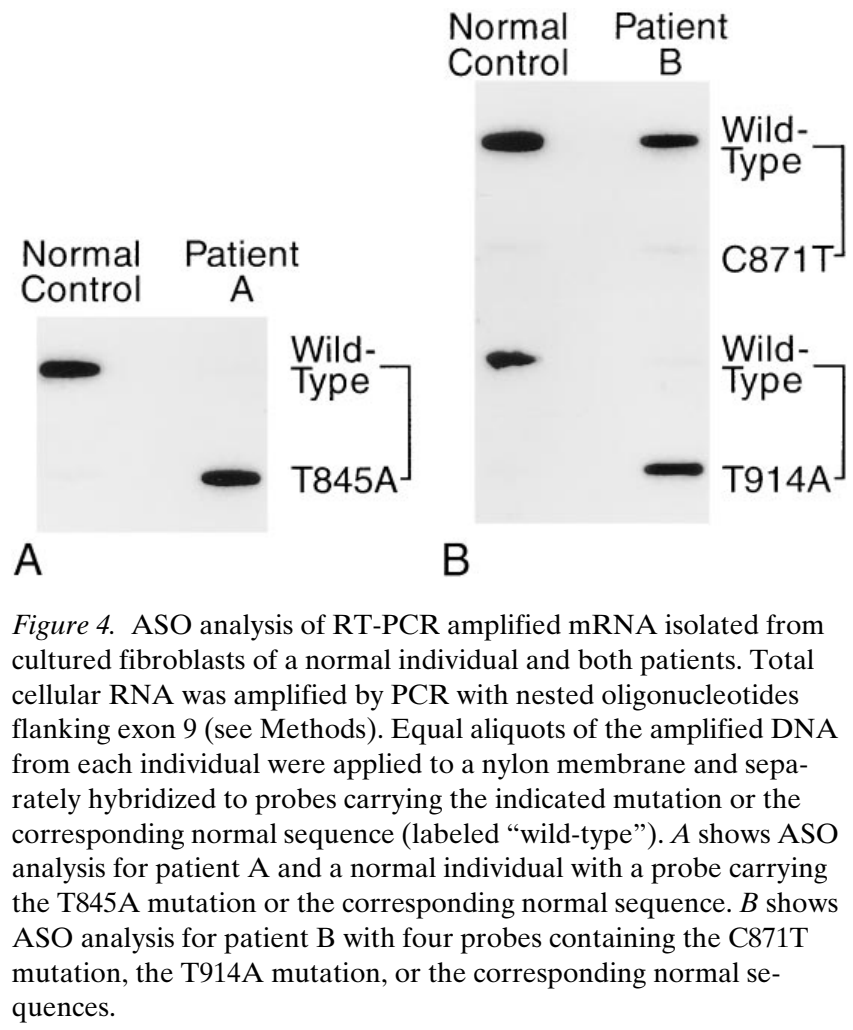

against the $\alpha$-subunit LCHAD domain and the $\beta$-subunit. Formation of TFP heteroctameric complex is essential for stable expression of both $\alpha$ - and $\beta$-subunits (11). Therefore, by measuring $\beta$-subunit expression, the amount of stable TFP complex generated can be assessed. Western blot with $\beta$-subunit antibody revealed stable expression of $\beta$-subunit, a surrogate measure of TFP, at levels comparable with the control in protein extracts derived from patient A's fibroblasts (Fig. 5). In three different experiments, densitometric scanning revealed mean $\beta$-subunit expression of $73 \%$ of control. Thus, the $\alpha$-subunit missense mutation slightly altered expression of the mutant TFP complex. In contrast, $\beta$-subunit (TFP protein) expression in extracts from patient B's fibroblasts was significantly reduced (Fig. 5). Quantification by densitometric scanning $(n=3)$ showed subunit expression of $28 \%$ of control. This result is consistent with the $\alpha$-subunit mRNA expression results outlined above, suggesting that the premature termination mutation mRNA and protein are not expressed at all. Decreases in $\alpha$-subunit protein expression paralleled those of the $\beta$-subunit.

If the missense mutant $\alpha$-subunits were incorporated into TFP equally as well as normal $\alpha$-subunit protein, we would have predicted that $\beta$-subunit expression would be $100 \%$ of control in patient $\mathrm{A}$ and $50 \%$ of control in patient $\mathrm{B}$. Because the observed levels of $\beta$-subunit are less $(73 \%$ and $28 \%$, respectively), our results suggest that these missense mutations alter TFP complex formation or stability to some extent.

\section{Discussion}

The slowly progressive myopathy and sensorimotor polyneuropathy manifested by the two patients in this study represent a unique fatty acid oxidation disorder phenotype which we have shown is associated with TFP deficiency by biochemical criteria (Table I). As compared with other TFP- and LCHAD-deficient patients, these two children had relatively late onset of

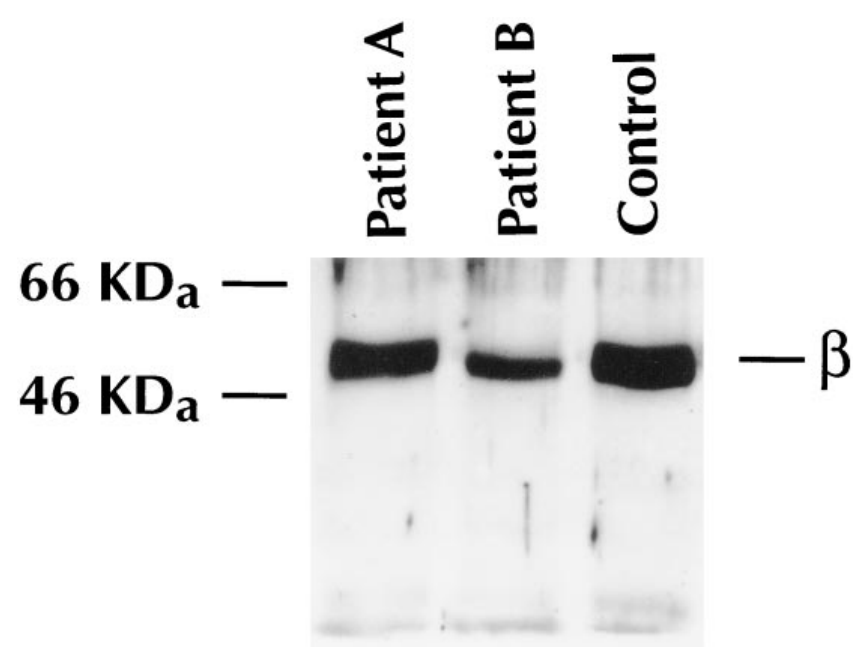

Figure 5. Immunoblot analysis of TFP $\beta$-subunit expression in protein extracts of fibroblasts from both patients. $50 \mu \mathrm{g}$ of protein extract from cultured fibroblasts of the patients (lanes $A$ and $B$ ) and a normal individual were analyzed as described in the Methods section and probed with $\beta$-subunit antiserum. This and two similar immunoblots were scanned by densitometry to quantify expression. 
disease and a milder course. The main result of our molecular genetic analysis is that both patients have missense mutations in exon 9 of the $\alpha$-subunit that are expressed in fibroblast mRNA and protein. Mutations in $\alpha$-subunit exon 9 have not been reported in other patients with isolated LCHAD or TFP deficiency $(3,5,6,8-14)$. The molecular defects causing TFP deficiency have been documented in only five other patients $(5,10,11)$, all of whom lacked neuromuscular manifestations similar to those described here, although clinical data in several are scant $(5,11)$. One TFP-deficient patient with cardiomyopathy and sudden death (10) has two $\alpha$-subunit donor splice site mutations, causing missplicing and no TFP protein expression. The other patients had $\beta$-subunit missense (D263G, R61H, R247H, R411K) or missplicing (T777Ins) mutations. All of these mutations were associated with inefficient TFP complex formation and failure to detect immunoreactive $\alpha$ - and $\beta$-subunits. Thus, the $\alpha$-subunit exon 9 mutations reported here are the first $\alpha$-subunit missense mutations causing complete TFP deficiency to be reported. We have identified the $\alpha$-subunit mutations in 24 patients with isolated LCHAD deficiency and 4 with complete TFP deficiency (Ibdah, J.A., and A.W. Strauss, unpublished results). None of these 28 patients exhibited the neuromuscular phenotype, and none had $\alpha$-subunit exon 9 mutations. Thus, the association shown here between $\alpha$-subunit exon 9 mutations and the neuromuscular phenotype is striking and suggests a novel genotype-phenotype correlation in TFP deficiency.

Exon 9 of the $\alpha$-subunit gene encodes a region in the mature $\alpha$-subunit protein that is not homologous to other enoylCoA hydratase or 3-hydroxyacyl-CoA dehydrogenase proteins. For this reason, the region has been described as a linker domain (4), and its function is not known. Potential roles of this domain might include binding to the inner mitochondrial membrane, binding or transfer of long-chain fatty acid substrates, or interaction with the $\beta$-subunit and formation of the stable TFP complex. Alternatively, mutations in this region might affect the overall three-dimensional TFP structure sufficiently to cause loss of enzyme activity.

Although the three exon 9 mutant alleles in our two patients result in TFP deficiency (as measured in fibroblast extracts), the mechanisms leading to lack of enzymatic activity are somewhat different. In patient $\mathrm{B}$, the splice site mutation is not expressed at the mRNA level, as expected from previous reports that attribute the decrease in cytoplasmic mutant mRNA level to rapid degradation of nuclear mRNA or pre-mRNA carrying premature termination codons (27). This nonsense codon-mediated RNA degradation occurs via trans-acting RNA binding proteins expressed in both yeast and mammals (28). This precursor mRNA degradation would account for our observed reduction in $\alpha$-subunit mRNA and a $50 \%$ decrease in TFP protein.

However, quantification of the protein expression shows that the missense mutations in both patients are expressed at lower levels than would be expected if each mutant protein were stably incorporated into TFP complex. In patient B, the I269N missense mutation, although allowing stable $\alpha$-subunit mRNA expression, results in reduced detectable $\alpha$ - and $\beta$-subunit protein in these cultured cells $(28 \%$ vs. the expected $50 \%$ ). Ushikubo and co-workers (11) demonstrated that formation of the TFP complex is essential for stabilization of both subunits. That is, lack of stable expression or mitochondrial in- corporation of $\alpha$-subunit protein would result in rapid degradation of the $\beta$-subunit as well, causing complete TFP deficiency. From our results, it seems likely that the I269N mutation disrupts TFP complex formation, either by slowing complex formation with concomitant rapid degradation of both $\alpha$ - and $\beta$-subunits or by accelerating degradation of the intact complex. By analogy to other missense mutations in another fatty acid oxidation enzyme, medium chain acyl-CoA dehydrogenase, the first mechanism seems more likely (29).

Both TFP subunits in patient A are expressed relatively well ( $73 \%$ of control), as demonstrated by Western blot analysis. Nonetheless, this modest reduction in protein expression also suggests that the V246D mutation alters TFP turnover. Even though significant $\alpha$ - and $\beta$-subunit antigen is expressed in both patients, long-chain 3-keto-acyl-CoA thiolase activity is reduced by $95 \%$ in fibroblasts (Table I). We speculate that altered TFP complex turnover is consistent with disruption of structure sufficient to reduce enzyme activities. Previous reports $(5,11)$ document that $\beta$-subunit missense mutations do alter TFP formation, turnover, and abolish enzyme activity. In the absence of the TFP crystallographic structure and analysis of detailed TFP structure-function relationships, it is difficult to explain with certainty the mechanism by which these exon 9 mutations produce the neuromuscular phenotype. An intriguing possibility is that exon 9 may result in this distinct biochemical phenotype secondary to accumulation of long-chain fatty acid metabolites that may be particularly toxic to both muscle and neuronal plasma or mitochondrial membranes, causing cell death. Mitochondria are central in apoptosis (30). This membranotoxic effect may be ameliorated by prednisone treatment, thereby accounting for the significant therapeutic response in one of the patients. Another possibility is that these metabolites or dying cells induce inflammation in muscle, as occurs in muscular dystrophies, and that the steroid response is secondary to reduction of inflammatory damage (31).

Our results are consistent with the concept that the relatively mild biochemical phenotype in these two patients with TFP deficiency occurs because some stable expression of TFP with the missense mutations occurs. Sufficient expression may allow survival to an age that neuromuscular manifestations become obvious. This may be similar to individuals with longer term survival and another fatty acid oxidation defect, very long-chain acyl-CoA dehydrogenase (VLCAD) deficiency, who develop recurrent myopathic episodes in later life (32). However, these VLCAD-deficient patients do not have polyneuropathy.

The data presented in this report suggest a novel genotypephenotype correlation in a mitochondrial fatty acid $\beta$-oxidation defect. Mutations in exon 9 of the $\alpha$-subunit of the mitochondrial TFP result in complete TFP deficiency and a unique phenotype characterized by a slowly progressive sensorimotor polyneuropathy and limb-girdle myopathy with recurrent episodes of myoglobinuria.

\section{Acknowledgments}

This study was supported by a grant from the National Institutes of Health (AM20407). J. Ibdah was supported by an institutional training grant (5T32 DK07130) from the National Institutes of Health. I. Tein was supported by a grant from the Physicians' Services Incorporated Foundation of Ontario. 


\section{References}

1. Uchida, Y., K. Izai, T. Orii, and T. Hashimoto. 1992. Novel fatty acid $\beta$-oxidation enzymes in rat liver mitochondria. J. Biol. Chem. 267:1034-1041.

2. Jackson, S., R.S. Kler, K. Barlett, H. Briggs, L.A. Bindoff, M. Pourfarzam, D. Gardner-Medwin, and D.M. Turnbull. 1992. Combined enzyme defect of mitochondrial fatty acid oxidation. J. Clin. Invest. 90:1219-1225.

3. Sims, H.F., J.C. Brackett, C.K. Powell, W.R. Treem, D.E. Hale, M.J. Bennett, B. Gibson, S. Shapiro, and A.W. Strauss. 1995. The molecular basis of pediatric long chain 3-hydroxyacyl-CoA dehydrogenase deficiency associated with maternal acute fatty liver of pregnancy. Proc. Natl. Acad. Sci. USA. 92: 841-845

4. Kamijo, T., A. Aoyama, A. Komiyama, and T. Hashimoto. 1994. Structural analysis of cDNAs for subunits of human mitochondrial fatty acid $\beta$-oxidation trifunctional protein. Biochem. Biophys. Res. Commun. 199:818-825.

5. Orii, K.E., T. Aoyama, K. Wakui, Y. Fukushima, H. Miyajima, S. Yamaguchi, T. Orii, N. Kondo, and T. Hashimoto. 1997. Genomic and mutational analysis of the mitochondrial trifunctional protein $\beta$-subunit (HADHB) gene in patients with trifunctional protein deficiency. Hum. Mol. Genet. 6:1215-1224.

6. IJlst, L., J.P.N. Ruiter, J.M.N. Hoovers, M.E. Jakobs, and R.J.A. Wanders. 1996. Common missense mutation G1528C in long-chain 3-hydroxyacylCoA dehydrogenase deficiency. J. Clin. Invest. 98:1028-1033.

7. Wanders, R.J.A., M. Duran, L. IJlst, J.P. de Jager, A.H. van Gennip, C. Jakobs, L. Dorland, and F.J. van Sprang. 1989. Sudden infant death and longchain 3-hydroxyacyl-CoA dehydrogenase. Lancet. 2:52-53.

8. Hagenfeldt, N., N. Venizelos, and U. Döbeln. 1995. Clinical and biochemical presentation of long chain 3-hydroxyacyl-CoA dehydrogenase deficiency. J. Inherit. Metab. Dis. 18:245-248.

9. Kamijo, T., R.J.A. Wanders, J.-M. Saudubray, T. Aoyama, A. Komiyama, and T. Hashimoto. 1994. Mitochondrial trifunctional protein deficiency. J. Clin. Invest. 93:1740-1747.

10. Brackett, J.C., H.F. Sims, P. Rinaldo, S. Shapiro, C.K. Powell, M.J. Bennett, and A.W. Strauss. 1995. Two $\alpha$-subunit donor splice site mutations cause human trifunctional protein deficiency. J. Clin. Invest. 95:2076-2082.

11. Ushikubo, S., T. Aoyama, T. Kamijo, R.J.A. Wanders, P. Rinaldo, J. Vockley, and T. Hashimoto. 1996. Molecular characterization of mitochondrial trifunctional protein deficiency: formation of the enzyme complex is important for stabilization of both $\alpha$ - and $\beta$-subunits. Am. J. Hum. Genet. 58:979-988.

12. Isaacs, J.D., H.F. Sims, C.K. Powell, M.J. Bennett, D.E. Hale, W.R. Treem, and A.W. Strauss. 1996. Maternal acute fatty liver of pregnancy associated with fetal trifunctional protein deficiency. Pediatr. Res. 40:393-398.

13. IJlst, L., R.J.A. Wanders, S. Ushikubo, T. Kamijo, and T. Hashimoto. 1994. Molecular basis of long-chain 3-hydroxyacyl-CoA dehydrogenase deficiency: identification of the major disease-causing mutation in the $\alpha$-subunit of the mitochondrial trifunctional protein. Biochem. Biophys. Acta. 1215:347-350.

14. IJlst, L., S. Ushikubo, T. Kamijo, T. Hashimoto, J.P.N. Ruiter, J.B.C. de Klerk, and R.J.A. Wanders. 1995. Long-chain 3-hydroxyacyl-CoA dehydrogenase deficiency: high frequency of the G1528C mutation with no apparent correlation with the clinical phenotype. J. Inherit. Metab. Dis. 18:241-244.

15. Dionisi-Vici, C., B. Garavaglia, A.B. Burlina, E. Bertini, I. Saponara, G. Sabetta, and F. Taroni. 1996. Hypoparathyroidism in mitochondrial trifunctional protein deficiency. J. Pediatr. 129:159-162.

16. Tein, I., E.J. Donner, D.E. Hale, and E.G. Murphy. 1995. Clinical and neurophysiologic response of myopathy and neuropathy in long-chain L-3-hydroxyacyl-CoA dehydrogenase deficiency to oral prednisone. Pediatr.
Neurol. 12:68-76.

17. Dionisi-Vici, C., A.B. Burlina, E. Bertini, C. Bachmann, M.R.M. Mazziotta, F. Zacchello, G. Sabetta, and D.E. Hale. 1991. Progressive neuropathy and recurrent myoglobinuria in a child with long-chain 3-hydroxyacyl-CoA dehydrogenase deficiency. J. Pediatr. 118:744-746.

18. Bertini, E., C. Dionisi-Vici, B. Garavaglia, A.B. Burlina, M. Sabatelli, M. Rimoldi, A. Bartuli, G. Sabetta, and S. DiDonato. 1992. Peripheral sensorymotor polyneuropathy, pigmentary retinopathy, and fatal cardiomyopathy in long-chain 3-hydroxy-acyl-CoA dehydrogenase deficiency. Eur. J. Pediatr. 151: 121-126.

19. Poll-The, B.T., J.P. Bonnefont, H. Ogier, C. Charpentier, A. Pelet, J.M. Le Fur, C. Jakobs, R.M. Kok, M. Divry, J. Scotto, and J.-M. Saudubray. 1988 Familial hypoketotic hypoglycemia associated with peripheral neuropathy, pigmentary retinopathy and C6-C14 hydroxydicarboxylic aciduria. A new defect in fatty acid oxidation? J. Inherit. Metab. Dis. 11:183-185.

20. Wanders, R.J.A., L. IJlst, F. Poggi, J.P. Bonnefont, A. Munnich, M. Brivet, D. Rabier, and J.-M. Saudubray. 1992. Human trifunctional protein deficiency. Biochem. Biophys. Res. Commun. 188:1139-1145.

21. Ausubel, F.M., R.E. Kingston, D.D. Moore, J.G. Seidman, J.A. Smith, and K. Struhl. 1994. Current Protocols in Molecular Biology. Wiley, New York. 1.7.1-1.7.15.

22. Chomczynski, P., and N. Sacchi. 1987. Single-step method of RNA isolation by acid guanidinium thiocyanate-phenol-chloroform extraction. Anal. Biochem. 162:156-159.

23. Orita, M., H. Iwahana, H. Kanazawa, K. Hayashi, and T. Sekiya. 1989. Detection of polymorphisms of human DNA by gel electrophoresis as singlestrand conformation polymorphisms. Proc. Natl. Acad. Sci. USA. 86:2766-2770.

24. Brackett, J.C., H.F. Sims, R.D. Steiner, M. Nunge, E.M. Zimmerman, B. deMartinville, P. Rinaldo, and A.W. Strauss. 1994. J. Clin. Invest. 94:1477-1483. 25. Laemmli, U.K. 1970. Cleavage of structural proteins during the assembly of the head of bacteriophage T4. Nature. 227:680-685.

26. Jackson, S., K. Bartlett, J. Land, E.R. Moxon, R.J. Pollitt, J.V. Leonard, and D.M. Turnbull. 1991. Long chain 3-hydroxyacyl-CoA dehydrogenase deficiency. Pediatr. Res. 29:406-411.

27. Aoufouchi, S., J. Yelamos, and C. Milstein. 1996. Nonsense mutations inhibit RNA splicing in a cell-free system: recognition of mutant codons independent of protein synthesis. Cell. 85:415-422.

28. Perlick, H.A., S.M. Medghalchi, F.A. Spencer, R.J. Kendzior, and H.C. Dietz. 1996. Mammalian orthologues of a yeast regulator of nonsense transcript stability. Proc. Natl. Acad. Sci. USA. 93:10928-10932.

29. Bross, P., C. Jespersen, T.G. Jensen, B.S. Andresen, M.J. Kristensen, V. Winter, A. Nancy, F. Kräutle, S. Ghisla, L. Bolund, J.-J.P. Kim, and N. Gregersen. 1995. Effects of two mutations in medium chain acyl-CoA dehydrogenase (MCAD)-deficient patients on folding, oligomer assembly, and stability of MCAD enzyme. J. Biol. Chem. 270:10284-10290.

30. Jürgensmeier, S.M., Z. Xie, Q. Deveraux, L. Ellerby, D. Bredesen, and J.C. Reed. 1998. Bax directly induces release of cytochrome $\mathrm{c}$ from isolated mitochondria. Proc. Natl. Acad. Sci. USA. 95:4997-5002.

31. Spencer, M.J., C.M. Walsh, K.A. Dorshkind, E.M. Rodriguez, and J.G. Tidball. Myonuclear apoptosis in dystrophic mdx muscle occurs by perforinmediated cytotoxicity. J. Clin. Invest. 99:2745-2751.

32. Vianey-Saban, C., P. Divry, M. Brivet, M. Nada, M.-T. Zabot, M. Mathieu, and C. Roe. 1998. Mitochondrial very-long-chain acyl-CoA dehydrogenase deficiency: clinical characteristics and diagnostic considerations in 30 patients. Clin. Chem. Acta. 269:43-62. 